\title{
Outcomes Associated with Treatment of Chronic Pain with Tapentadol Compared with Morphine and Oxycodone: A UK Primary Care Observational Study
}

\author{
Christopher Ll. Morgan (D) · Sara Jenkins-Jones • Craig Currie • \\ Garth Baxter
}

Received: January 16, 2019 / Published online: April 8, 2019

(c) The Author(s) 2019

\section{ABSTRACT}

Introduction: This study compared adverse outcomes and resource use for patients with a diagnosis of pain treated with tapentadol prolonged-release (PR) versus those treated with morphine controlled-release (CR) and oxycodone CR.

Methods: Data were sourced from the Clinical Practice Research Datalink (CPRD), a database derived from UK primary care. Patients prescribed tapentadol PR between May 2011 and December 2016 were selected and matched to two groups of controls treated with either morphine CR or oxycodone CR on gender, age, pain duration, pain site, pain aetiology, Charlson index and prior analgesia. Times to first adverse event (constipation or nausea/vomiting) were compared within a Cox proportional hazards model. Rates of primary care contacts,

Enhanced Digital Features To view enhanced digital features for this article go to https://doi.org/10.6084/ m9.figshare.7830764.

Electronic supplementary material The online version of this article (https://doi.org/10.1007/s12325019-00932-7) contains supplementary material, which is available to authorized users.

C. Ll. Morgan $(\varangle) \cdot$ S. Jenkins-Jones · C. Currie Pharmatelligence, Cardiff, UK

e-mail: Chris.morgan@pharmatelligence.co.uk

G. Baxter

Grünenthal Ltd, Stokenchurch, UK accident and emergency contacts and, for a subset of patients linked to Hospital Episode Statistics (HES), inpatient admissions and outpatient contacts were compared using incidence rate ratios (IRRs) derived from Poisson regression.

Results: A total of 1907 patients prescribed tapentadol PR were identified and 1791 (93.9\%) had a pain diagnosis. Of these 1246 (65.3\%) were matched to morphine controls and 829 (43.4\%) to oxycodone controls. Compared to controls, gastrointestinal adverse events with tapentadol PR treatment were reduced; $\mathrm{aHR}=$ $0.532(0.402-0.703 ; p<0.001)$ versus morphine CR and $0.517(0.363-0.735 ; p<0.001)$ versus oxycodone $\mathrm{CR}$. Compared with morphine CR, primary care contacts [IRR $=0.831$ (0.802-0.861)], accident and emergency attendance $[0.739(0.572-0.951)]$, outpatient contacts $[0.917(0.851-0.989)]$ and inpatients contacts $[0.789(0.664-0.938)]$ were reduced. For oxycodone, the respective figures were 0.735 (0.703-0.768), 0.971 (0.699-1.352), 0.877 (0.799-0.962) and 0.748 (0.601-0.932).

Conclusion: Tapentadol PR was associated with significantly fewer adverse gastrointestinal events than morphine CR and oxycodone CR in patients with a diagnosis of pain. There was also significantly reduced primary and secondary care resource use. As with all observational studies, potential bias due to residual confounding and confounding by indication should be considered. 
Funding: Grünenthal Ltd.

Keywords: Constipation; Gastrointestinal adverse events; Health service utilisation; Morphine; Nausea; Oxycodone; Pain; Tapentadol

\section{INTRODUCTION}

Pain is defined as "an unpleasant sensory and emotional experience associated with actual or potential tissue damage, or described in terms of such damage" [1]. Chronic pain is that which has a duration greater than 12 weeks. In the UK it is estimated that there are 7.8 million patients suffering from chronic pain, 2.8 million of whom would describe their pain as severe [2]. From the perspective of the individual, chronic pain can have a major impact on quality of life: $50 \%$ of sufferers report an effect on usual day to day activities, whilst $65 \%$ report problems with sleep patterns and 50\% report depression [3].

There is a recognised treatment pathway for chronic pain based upon the World Health Organization (WHO) cancer pain ladder, whereby patients progress from non-opioids such as paracetamol and non-steroidal anti-inflammatory drugs (NSAIDs) to weak opioids and finally strong opioids. Tapentadol is a centrally acting analgesic which has two mechanisms of action, namely $\mu$-opioid receptor agonism (MOR) and noradrenaline reuptake inhibition (NRI) [4]. Tapentadol, marketed as Palexia in the UK, is currently available in two forms: (1) immediate release preparations (Palexia film-coated tablets and Palexia oral solution), (2) prolonged-release tablets (Palexia SR). Palexia SR is used in the management of severe chronic pain in adults, which can be adequately managed only with opioid analgesics.

Morphine and oxycodone are alternative agents for the treatment of severe chronic pain. Both of these products are well established in the UK and are relatively inexpensive compared with tapentadol. However, both are associated with tolerability issues such as constipation, nausea and vomiting $[5,6]$. In phase III trials, tapentadol PR demonstrated comparable analgesic efficacy (based on patients' self-reported pain intensity [11-point NRS] and global impression of change [PGIC] scale), superior gastrointestinal tolerability (constipation, nausea and vomiting) and significantly fewer discontinuations compared with oxycodone CR $[7,8]$. Patients were able to stay on tapentadol PR for a median of 118 days compared with 39 days with oxycodone CR [7]. Superior quality of life outcomes have also been reported by patients administered tapentadol PR, when compared with those in the oxycodone CR arm [7]. In trials comparing tapentadol PR with morphine CR for severe chronic tumour-related pain, incidences of gastrointestinal adverse events favour the tapentadol PR group $[9,10]$.

The purpose of this study was to investigate whether the rates of adverse events observed in clinical trials comparing patients treated with tapentadol PR with those treated with morphine or oxycodone were observed within a real-world setting. In addition, we wished to consider if there were differences in health service utilisation and associated costs.

\section{METHODS}

\section{Data Source}

Patients were selected from the Clinical Practice Research Datalink (CPRD), formerly the General Practice Research Database (GPRD). CPRD is a longitudinal, anonymised research database derived from nearly 700 primary care practices in the UK. CPRD is funded by the National Health Service (NHS) National Institute for Health Research (NIHR) and the Medicines and Healthcare products Regulatory Agency (MHRA) and operates on a non-profit basis. Ethical approval for studies using the CPRD was granted by the Trent Multicentre Research Ethics Committee (reference 05/MRE04/87). Individual studies also require approval by the Independent Scientific Advisory Committee (ISAC). ISAC approval was granted for this study on 14 May 2014 (ISAC Protocol 14_092R). Informed consent was not required due to the anonymity of the CPRD data.

Data within CPRD are collected as part of the day-to-day administration of the healthcare 
system. The primary care data set (CPRD GOLD) comprises data on demographics, diagnoses, hospital referrals, prescriptions emanating in primary care, and other aspects of patient care. Approximately $60 \%$ of practices participate in a linkage scheme, by which their patient records are linked to other data sources, including the Hospital Episode Statistics (HES) data set, which provides data on all inpatient and outpatient contacts occurring within NHS hospitals in England, and the Office for National Statistics (ONS) mortality data set. Diagnostic information in the CPRD primary care data set is recorded using the Read code classification, a UK primary care practice standard. HES inpatient data are recorded using the ICD-10 classification.

Data quality is ascribed in CPRD by flags applied at a practice and patient level. Practices are deemed to be up-to-standard (UTS) when the data recorded for each practice in terms of death, prescribing and referral rates are within the range expected on the basis of practice size and demographics. Further quality control is applied to individual patients who are classified as acceptable if they have a valid coding for gender, have a valid birth year with no prior activity recorded before this date and also an age of below 115 at last data collection point. They must also be permanently registered at the practice.

\section{Patient Selection}

Patients were selected from May 2011 (when tapentadol was first available in the UK) to June 2016 (the most recent CPRD GOLD release). All patients prescribed tapentadol PR were extracted from the CPRD database using product codes recorded within CPRD and the date of first prescription of tapentadol PR defined the study index date. Prescriptions for analgesia (non-opioids, adjuvant co-analgesics, weak opioids and strong opioids) before index date were linked, allowing a maximum of 90 days between prescriptions, to define continuous pain episodes. Date of first prescription in the pain episode defined the start of the pain episode. Underlying pain diagnosis was ascertained from relevant Read codes, with the nearest relevant diagnosis to the index date (first prescription for tapentadol) used to classify the pain type. Baseline characteristics and concomitant medications for these patients were extracted and a prior pain pathway estimated on the basis of preceding prescriptions of nonopioids, weak opioids and strong opioids for pain management.

For comparative controls, all patients with a prescription for oxycodone or morphine CR (May 2011-June 2016 inclusive) were extracted. Pain pathways were estimated using the same method as for the tapentadol PR patients. Those patients with a first prescription of either oxycodone CR or morphine CR within their pain episode since 2011 were flagged as potential controls. Controls were matched directly at a ratio of 1:1 for each member of the tapentadol PR cohort on the basis of gender, age ( \pm 5 years), pain duration, that is duration of pain episode ( \pm 180 days), pain site and aetiology, same maximum stage of the pain pathway and HES linkage scheme status (HES-eligible, HES-ineligible). Cases and the oxycodone CR and morphine CR control sets were mutually exclusive, that is patients could only appear in one analytical cohort.

\section{Outcomes}

\section{Adverse Gastrointestinal Events}

The primary outcome was time to first subsequent constipation or nausea/vomiting event or a combined endpoint of the two. Adverse events were identified by diagnoses recorded within either the CPRD primary care or HES secondary care data sources coded using the Read or ICD10 classifications respectively. Patients were followed from index date to the earliest of date of first recorded event, date of discontinuation (that is date of last index drug prescription +40 days) or censorship. Censorship was defined as the earliest of end of pain episode, the patient's last contact date or the last data collection point for their particular practice. Crude rates of adverse events were calculated per person year. Time to event was compared using a Cox proportional hazards model 
adjusting for age, gender, Charlson index, pain episode duration, concomitant therapy (nonopioids, weak opioids or other strong opioids) and pharmacological adjuvant co-analgesia therapy. For each outcome in the Cox model, the adjusted hazard ratio was provided with 95\% confidence intervals.

\section{Secondary Outcomes}

The secondary outcomes were rates of resource utilisation. These were primary care and accident and emergency contacts identified in the primary care database and outpatient and inpatient contacts identified for the subset of patients linkable to HES secondary care sources. Crude utilisation rates were calculated and rates of activity compared between different cases and controls using Poisson regression models adjusting for age, gender, Charlson index and primary care contacts in the 12 months prior to baseline, duration of pain episode and adjuvant co-analgesia therapy.

All healthcare contacts were costed. Primary care contacts with a healthcare professional were identified and classified by consultation type (e.g. surgery appointment, clinic, home visit, telephone consultation) and staff type (e.g. general practitioner (GP), practice nurse, district nurse) and then assigned a unit cost as listed in the Unit Cost of Health and Social Care 2016 [11]. Data from hospital inpatient admissions and outpatient attendances recorded in HES were processed into healthcare resource groups (HRGs) using the HRG-4 grouper [12]. The allocated HRGs were linked to the national tariff [13]. As there were insufficient data available within the primary care data set to accurately apply HRGs to the accident and emergency data, a mean accident and emergency cost was applied derived from the tariffs [13].

\section{Therapy Changes}

Concomitant medication (non-opioids, weak opioids, strong opioids and adjuvant co-analgesic therapy e.g. antidepressants, antiepileptics, muscle relaxants) prescribed at baseline were defined as those where prescription date was between \pm 30 days of index date. Patients with either baseline medication prescribed or a subsequent prescription within the therapy episode were defined as receiving concomitant therapy. The proportion of patients with therapy added to their regimen was described. Time to first additional concomitant therapy following index date was compared between groups using a Cox proportional hazards model adjusting for age, gender and Charlson index.

\section{Sensitivity Analyses}

Dose information was not available for all patients. Where these data were available, sensitivity analyses were performed for the gastrointestinal adverse event and resource utilisation analyses including therapy starting dose as a covariate.

\section{RESULTS}

\section{Identified Patients}

A total of 1907 patients with a first prescription of tapentadol PR were identified. Baseline characteristics are shown in Table 1 . Of those identified patients, 1206 (63.2\%) were female. Mean age was 56.2 (sd 14.8) for male patients and 57.0 (sd 16.2) for female patients. The mean duration of the estimated pain episode was 3.5 (sd 4.5) years. A majority of patients (51.0\%) had progressed through the pain pathway via non-opioids, weak opioids and strong opioids. There was no underlying aetiology other than pain recorded in their patient records for 910 (47.7\%) patients. The most common underlying aetiology recorded was musculoskeletal recorded for $318(16.7 \%)$ patients. A total of 116 (6.0\%) tapentadol PR patients were excluded from the matching process as a result of either having no aetiology recorded at all (77 (4.0\%)) or having only depression recorded (39 (2.0\%)).

Following the application of the matching criteria, 1246 (65.3\%) tapentadol PR patients could be matched in the morphine CR comparison and $829(43.5 \%)$ could be matched in the oxycodone CR comparison. There were no significant differences between the cohorts at 


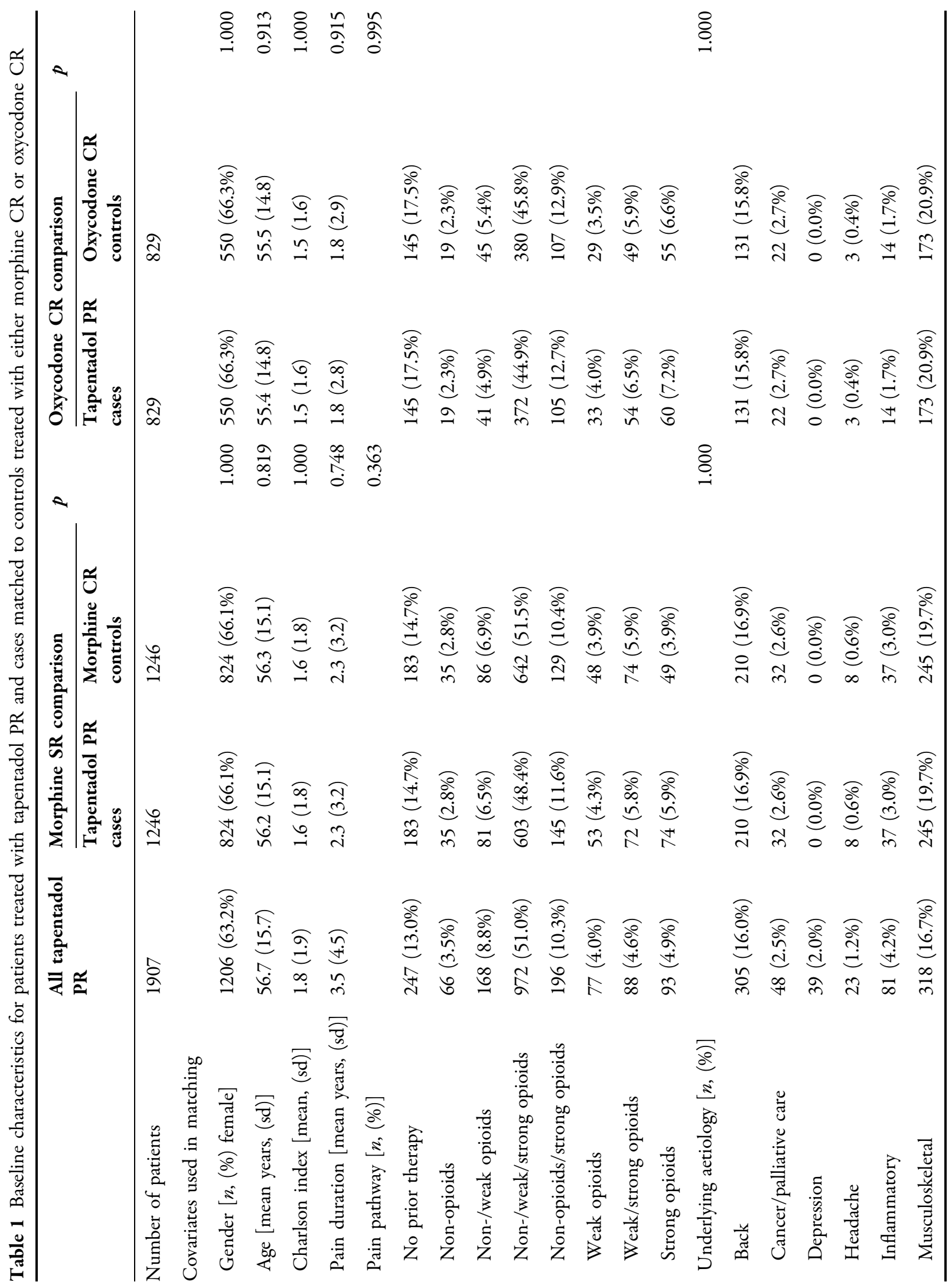




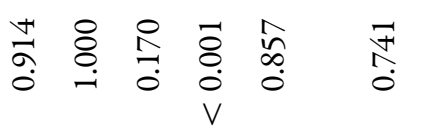

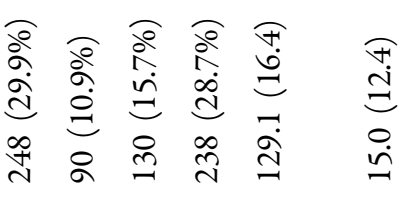

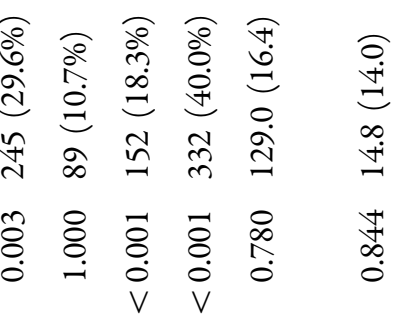

2

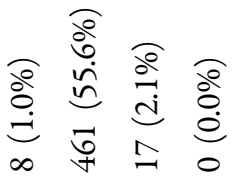

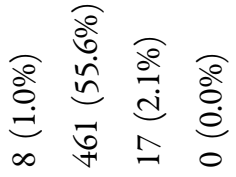

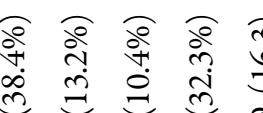

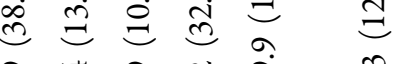

શ

苍

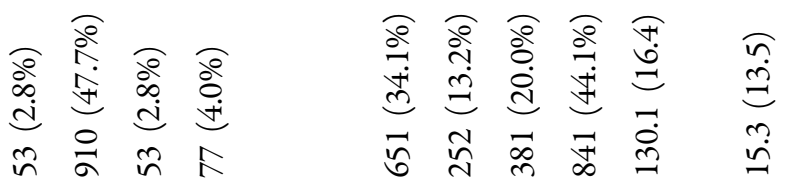

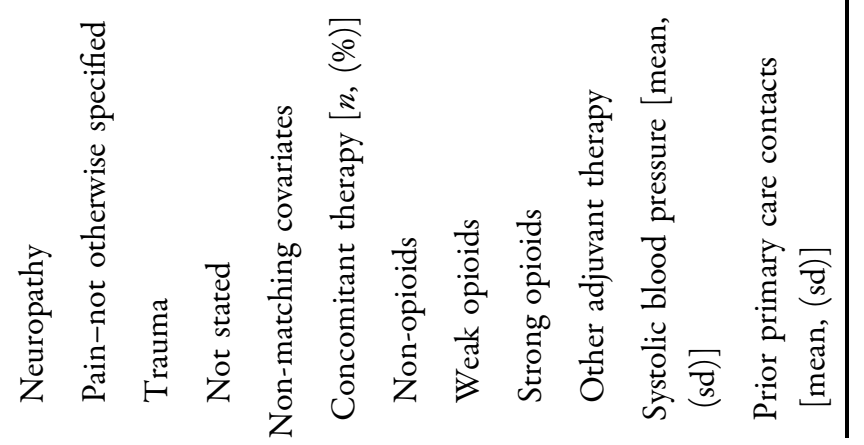




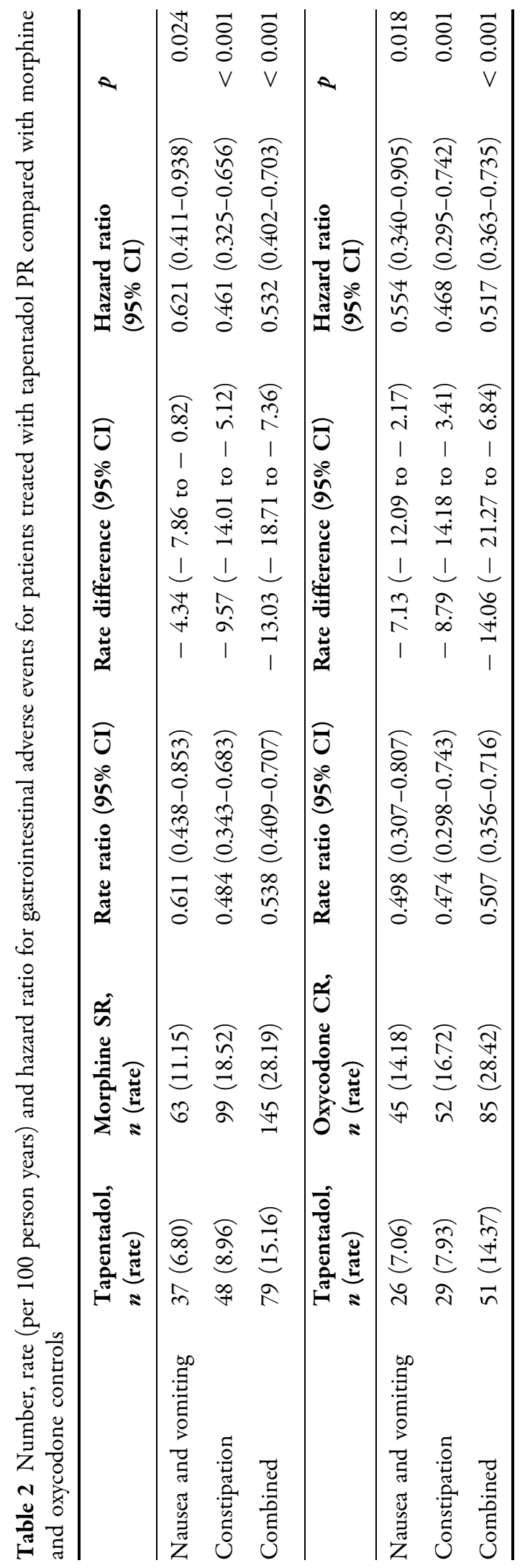




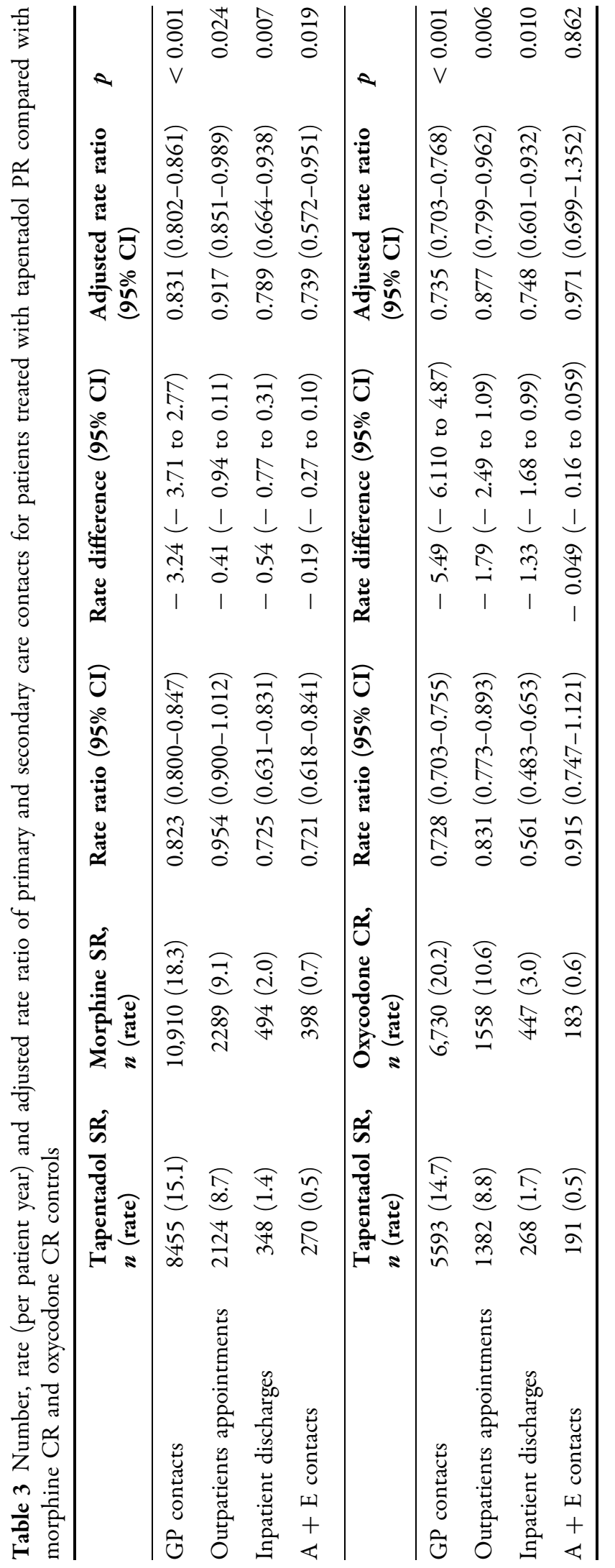


Table 4 Mean and median costs $(£)$ of primary and secondary care contacts for patients treated with tapentadol PR compared with morphine CR and oxycodone CR controls

\begin{tabular}{|c|c|c|c|c|c|}
\hline & \multicolumn{2}{|l|}{ Cases } & \multicolumn{2}{|l|}{ Controls } & \multirow[t]{2}{*}{$p$} \\
\hline & Mean (sd) & Median (IQR) & $\overline{M e a n}(\mathbf{s d})$ & Median (IQR) & \\
\hline \multicolumn{6}{|l|}{ Morphine SR comparison } \\
\hline GP contacts (per patient year) & $707(734)$ & $516(271-895)$ & $982(1191)$ & $676(356-1204)$ & $<0.001$ \\
\hline $\begin{array}{l}\text { Outpatients appointments (per } \\
\text { patient year) }\end{array}$ & $974(1915)$ & $536(0-1230)$ & $1278(2355)$ & $651.5(0-1491)$ & 0.013 \\
\hline $\begin{array}{l}\text { Inpatient discharges (per patient } \\
\text { year) }\end{array}$ & $2582(8480)$ & $0(0-1415)$ & $5741(14,068)$ & $0(0-4100)$ & $<0.001$ \\
\hline $\mathrm{A}+\mathrm{E}$ contacts (per patient year) & $95(491)$ & $0(0-0)$ & $117(393)$ & $0(0-0)$ & 0.002 \\
\hline Total costs & $4414(9157)$ & $1764(777-3841)$ & $8220(15,446)$ & $2504(1008-7723)$ & $<0.001$ \\
\hline \multicolumn{6}{|l|}{ Oxycodone CR comparison } \\
\hline GP contacts (per patient year) & $672(700)$ & $498(248-816)$ & $1029(1416)$ & $685(334-1317)$ & $<0.001$ \\
\hline $\begin{array}{l}\text { Outpatients appointments (per } \\
\text { patient year) }\end{array}$ & $939(2084)$ & $536(0-1141)$ & $1479(6586)$ & $633(0-1523)$ & 0.105 \\
\hline $\begin{array}{l}\text { Inpatient discharges (per patient } \\
\text { year) }\end{array}$ & $2341(7654)$ & $0(0-1193)$ & $4218(10,589)$ & $0(0-2446)$ & 0.081 \\
\hline $\mathrm{A}+\mathrm{E}$ contacts (per patient year) & $85(456)$ & $0(0-0)$ & $92(403)$ & $0(0-0)$ & 0.935 \\
\hline Total costs & $4080(8428)$ & $1652(698-3459)$ & $6860(13,123)$ & $2158(892-6755)$ & 0.002 \\
\hline
\end{tabular}

baseline other than for patterns of concomitant therapy. In the morphine CR comparison patients treated with tapentadol PR were less likely to be treated with non-opioids $(32.6 \%$ versus $38.4 \% ; p=0.003$ ) but more likely to be treated with both other strong opioids $(18.4 \%$ versus $10.4 \% ; p<0.001$ ) and adjuvant co-analgesia therapy $(40.9 \%$ versus $32.3 \% ; p<0.001)$. In the oxycodone CR comparison, tapentadol PR cases were more likely to be treated with other adjuvant co-analgesia therapy (40.0\% versus $28.7 \% ; p<0.001$ ).

\section{Treatment Exposure}

In the comparison with morphine $\mathrm{CR}$, median duration on treatment was 319 days (IQR 104-1658) for patients treated with tapentadol PR and 315 days (IQR 124-1639) for the those treated with morphine CR. In the oxycodone CR comparison, median duration on tapentadol
PR was 392 days (IQR 114-1658) versus 279 (IQR 90-unknown) for those treated with oxycodone CR.

In the comparison with morphine, the mean dosage was titrated from $145.9 \mathrm{mg}$ (sd 86.6) [morphine equivalent dose (MED) 58.4 (sd 34.6)] at therapy start to $194.8 \mathrm{mg}$ (sd $118.1 \mathrm{mg}$ ) [MED 77.9 (sd $47.2 \mathrm{mg}$ )] for final prescription for patients initiating tapentadol and from $34.4 \mathrm{mg}$ (sd 31.5) to $43.5 \mathrm{mg}$ (sd 42.3) for patients initiating morphine. In the oxycodone comparison, the dosage for patients treated with tapentadol increased from $142.4 \mathrm{mg}$ (sd 81.6) [MED 57.0 (sd $32.6 \mathrm{mg}$ )] to $191.2 \mathrm{mg}$ (sd 114.3) [MED $76.5 \mathrm{mg}$ (sd 45.7)] compared with $32.3 \mathrm{mg}$ (sd 31.4) [MED 64.6 (sd 62.8)] to $38.5 \mathrm{mg}$ (sd 35.7) [MED 77.0 (sd $71.4 \mathrm{mg}$ )] of oxycodone. Although lower than in controlled trials, the mean final total daily dose of tapentadol PR was comparable to that of oxycodone $\mathrm{CR}$ and higher than that of morphine $\mathrm{CR}$, based on the accepted 
Table 5 Concomitant therapy for patients treated with tapentadol PR compared with morphine CR and oxycodone CR controls

\begin{tabular}{lcccr}
\hline & $\begin{array}{l}\text { Case } \\
\text { Number (\%) }\end{array}$ & $\begin{array}{l}\text { Control } \\
\text { Number (\%) }\end{array}$ & Hazards ratio & $p$ \\
\hline Morphine comparison & $150(17.86 \%)$ & $202(26.34 \%)$ & $0.741(0.598-0.918)$ & 0.006 \\
Non-opioids & $45(4.16 \%)$ & $47(4.34 \%)$ & $1.124(0.744-1.700)$ & 0.579 \\
Weak opioids & $100(9.83 \%)$ & $77(6.89 \%)$ & $1.853(1.369-2.507)$ & $<0.001$ \\
Strong opioids & $258(35.01 \%)$ & $256(30.33 \%)$ & $1.424(1.195-1.698)$ & 0.017 \\
Adjuvant therapy & & & & $0.481(0.372-0.622)$ \\
Oxycodone comparison & $89(15.24 \%)$ & $172(29.60 \%)$ & $0.693(0.419-1.148)$ & 0.155 \\
Non-opioids & $26(3.51 \%)$ & $38(5.14 \%)$ & $0.914(0.658-1.268)$ & 0.589 \\
Weak opioids & $70(10.34 \%)$ & $79(11.30 \%)$ & $1.416(1.164-1.723)$ & 0.001 \\
Strong opioids & $178(35.81 \%)$ & $178(30.12 \%)$ & & \\
Adjuvant therapy & & & & \\
\hline
\end{tabular}

dose conversion ratios of approximately 5:1 and 2.5:1 for tapentadol PR to oxycodone CR and morphine CR respectively.

\section{Adverse Events}

There was a significantly reduced hazard ratio for all gastrointestinal adverse events favouring tapentadol PR versus both morphine $\mathrm{CR}$ and oxycodone $\mathrm{CR}$. The adjusted hazard ratios versus morphine CR were $0.621(0.411-0.938)$ for nausea and vomiting, $0.461(0.325-0.656)$ for constipation and $0.532(0.402-0.703)$ for the combined endpoint. For the comparison of tapentadol PR with oxycodone CR the respective hazard ratios were 0.554 (0.34-0.905), $0.468(0.295-0.742)$ and $0.517(0.363-0.735)$ (Table 2).

In a sensitivity analysis adjusting for baseline treatment dose, the association remained for all outcomes in both comparisons (Supplementary Table 1).

\section{Resource Use}

Resource use was reduced for patients treated with tapentadol PR compared with morphine
CR. Primary care contacts were 15.1 versus 18.3 per patient year; adjusted incidence ratio 0.831 (0.802-0.861). Respective figures for outpatient, inpatient and accident and emergency contacts were 8.7 versus 9.1 [0.917 (0.851-0.989)], 1.4 versus 2.0 [0.789 (0.664-0.938)] and 0.5 versus 0.7 [0.739 (0.572-0.951)].

Similar results were observed for the comparison with oxycodone $\mathrm{CR}$, though there was no significant difference in adjusted incidence ratio for accident and emergency contacts. The respective figures for primary care, outpatient, inpatient and accident and emergency contacts were 14.7 versus 20.2 [0.735 (0.703-0.768)], 8.8 versus 10.6 [0.877 (0.799-0.962)], 1.7 versus 3.0 [0.748 (0.601-0.932)] and 0.5 versus 0.6 [0.971 (0.699-1.352)], (Table 3).

The reduced resource use was reflected in associated costs. Median total costs were $£ 1764$ (IQR £777-3841; mean £4414) per patient year for those treated with tapentadol PR compared with $£ 2504$ (IQR £1008-7723; mean £8220) for those treated with morphine CR. The respective costs in the oxycodone comparison were $£ 1652$ (IQR £698-3459; mean £4080) versus £.2158 (IQR £892-6755; mean £6860) (Table 4).

In a sensitivity analysis adjusting for baseline treatment dose, the association remained for all 
outcomes in both comparisons (Supplementary Table 2).

\section{Concomitant Medication}

In both comparisons, patients treated with tapentadol PR had increased time to use of additional concomitant non-opioids during the therapy period: $\mathrm{HR}=0.741 \quad(95 \% \quad \mathrm{CI}$ 0.598-0.918) compared with patients treated with morphine $\mathrm{CR}$ and $\mathrm{HR}=0.481$ (95\% CI $0.372-0.622$ ) for those treated with oxycodone CR; whereas time to additional adjuvant therapy was reduced in tapentadol treated patients $\mathrm{HR}=1.424 \quad(95 \%$ CI 1.195-1.698) and $\mathrm{HR}=$ 1.416 (1.164-1.723) compared with those treated with morphine and oxycodone respectively. Time to additional use of strong opioids was also reduced for tapentadol patients in the morphine comparison [1.853 (1.369-2.507)]. There were no other significant differences in the additional use of other therapies (Table 5).

\section{DISCUSSION}

Within this observational study based on UK routine data, treatment with tapentadol PR was associated with significantly fewer adverse gastrointestinal events compared with either morphine CR or oxycodone CR controls. There were also significantly fewer primary and secondary care contacts and consequently significantly lower healthcare costs associated with tapentadol PR.

Compared with oxycodone CR, we reported an approximate halving of the hazard ratio for both nausea and vomiting [0.524 (0.323-0.851)] and constipation $[0.470(0.332-0.664)]$. The reduced rates of gastrointestinal adverse events that we report from this observational study support findings from clinical trial data. In a phase III trial of patients with chronic low back pain the incidence of gastrointestinal adverse events for patients treated with tapentadol PR versus oxycodone CR were $20.1 \%$ versus $34.5 \%$ for nausea, $13.8 \%$ versus $26.8 \%$ for constipation and $9.1 \%$ versus $19.2 \%$ for vomiting [14]. These results were similar for patients with chronic osteoarthritis of the knee, in which $21.5 \%$ versus $36.5 \%$ of patients reported nausea, $18.9 \%$ versus $36.8 \%$ of patients reported constipation and $5.2 \%$ versus $17.8 \%$ of patients reported vomiting on tapentadol PR compared with oxycodone CR treatment respectively [15]. In a trial of patients with chronic malignant tumour-related pain, tapentadol PR was also associated with a lower incidence of gastrointestinal adverse events than morphine (38.0\% versus $54.0 \%$ for constipation) [9].

Similar findings have been reported when comparing immediate release tapentadol and oxycodone. In a study of patients with endstage joint disease awaiting surgery the odds ratios for tapentadol versus oxycodone $10 \mathrm{mg}$ for nausea and vomiting were 0.21 (95\% CI $0.128-0.339$ ) and 0.32 (95\% CI 0.204-0.501) for tapentadol $50 \mathrm{mg}$ and tapentadol $75 \mathrm{mg}$ dosages respectively; for constipation the respective odds ratios were 0.13 (95\% CI 0.057-0.302) and 0.20 (95\% CI 0.098-0.398) [16]. Reduced rates of gastrointestinal adverse events have also been reported in patients following bunionectomy for immediate release tapentadol $(50 \mathrm{mg})$ versus oxycodone (10 mg) [17].

In addition to reduced adverse gastrointestinal events, we found all resource use and associated costs were significantly reduced for patients treated with tapentadol PR compared with both sets of controls with the sole exception of accident and emergency contacts in the comparison with oxycodone CR controls. This supports data from a US study of tapentadol immediate release compared with oxycodone which found a significant reduction in inpatient admissions (0.07 vs 0.10 ), inpatient costs (\$2900 vs \$4382) and outpatient costs (\$10,550 vs $\$ 11,084)$ in a 60 -day period post index [18].

Economic models based on tapentadol have also shown it to be cost-effective compared with both oxycodone and morphine. On the basis of the UK health system, tapentadol PR was shown to be dominant versus oxycodone CR achieving improved quality of life for lower cost [19]. Similar results were also observed in a model based on the Spanish system [20] and within a budget impact model based on a US health plan [21]. The reduction in costs was achieved by both reduced pharmacy costs, driven by lower daily average consumption, and reduction in 
health service utilisation due to fewer opioidinduced adverse events. In addition, it is known that opioid-induced constipation has a major impact on patient quality of life often leading to poor compliance and consequently suboptimal pain management [22].

As with all observational studies, potential bias due to issues of residual confounding and confounding by indication should be considered. We attempted to match patients on key demographic, diagnostic and treatment characteristics, but it is not possible within routine data to achieve the same balance of covariates as can be achieved by randomisation within a trial setting. As severity of pain is not systematically recorded in a consistent manner we could not match patients according to baseline severity nor assess and compare effectiveness between treatment groups. Whilst we matched on duration of pain episodes, and there was no significant difference in pain duration ( 2.3 years and 1.8 years in both arms for the morphine and oxycodone comparisons respectively), duration of pain episodes was not explicit in the data but was calculated by a proxy measure of consecutive prescriptions ( $\leq 90$ days) to define each episode. This may have omitted some episodes where patients used therapy less frequently possibly in response to symptomatic pain and also incorrectly constructed concurrent pain episodes from different acute events. In addition, underlying aetiology was not explicitly connected with the prescription of either tapentadol PR or the control arms. We therefore attributed the underlying aetiology of the pain episode to the nearest diagnosis to index date and there may be some ambiguity in this attribution. However, for a large number of cases no diagnosis other than pain itself was recorded. We were able to match $65.3 \%$ of tapentadol patients to a morphine control and $43.5 \%$ of patients to an oxycodone control. The generalisability of these results to the wider cohorts should therefore be considered.

Secondary care inpatient and outpatient data is known to be reasonably robust but accident and emergency data available through HES has historically been known to be incomplete in terms of the coverage of units providing data and also of poor quality. We therefore used letters received by the primary care practice from the accident and emergency department as a proxy. We acknowledge that this is likely to underestimate the number of contacts considerably but we have no reason to suspect that this would introduce a bias when comparing the relative rate of contacts between treatment arms.

\section{CONCLUSION}

To conclude, this study has shown that the reduced incidence of gastrointestinal adverse events associated with tapentadol PR reported in clinical trials is also observed within a realworld setting. In addition, there is a reduced resource utilisation and consequently cost associated with tapentadol PR-treated patients. Differences between cases and controls should, however, be considered when interpreting these results.

\section{ACKNOWLEDGEMENTS}

Funding. Sponsorship for this study, the article processing charges and the Open Access fee were funded by Grünenthal Ltd. All authors had full access to all of the data in this study and take complete responsibility for the integrity of the data and accuracy of the data analysis.

Authorship. All named authors meet the International Committee of Medical Journal Editors (ICMJE) criteria for authorship for this article, take responsibility for the integrity of the work as a whole, and have given their approval for this version to be published.

Disclosures. Christopher Morgan is employed by Pharmatelligence who received funding to perform this study from Grünenthal Ltd. Craig Currie is employed by Pharmatelligence who received funding to perform this study from Grünenthal Ltd. Sara-Jenkins Jones is employed by Pharmatelligence who received funding to perform this study from Grünenthal 
Ltd. Garth Baxter is an employee of Grünenthal Ltd.

Compliance with Ethics Guidelines. Ethical approval for studies using the CPRD was granted by the Trent Multicentre Research Ethics Committee (reference 05/MRE04/87). Individual studies also require approval by the Independent Scientific Advisory Committee (ISAC). ISAC approval was granted for this study on 14 May 2014 (ISAC Protocol 14_092R). Informed consent was not required due to the anonymity of the CPRD data.

Data Availability. The data sets analysed during the current study were used under licence from Clinical Practice Research Datalink (CPRD). Data for this study can be accessed via application to CPRD.

Open Access. This article is distributed under the terms of the Creative Commons Attribution-NonCommercial 4.0 International License (http://creativecommons.org/licenses/ by-nc/4.0/), which permits any noncommercial use, distribution, and reproduction in any medium, provided you give appropriate credit to the original author(s) and the source, provide a link to the Creative Commons license, and indicate if changes were made.

\section{REFERENCES}

1. Merskey H. Pain terms: a list with definitions and notes on usage recommended by the IASP subcommittee on taxonomy. Pain. 1979;6:249-52.

2. Breivik H, Collett B, Ventafridda V. Survey of chronic pain in Europe: prevalence, impact on daily life and treatment. Eur J Pain. 2006;10:287-333.

3. Donaldson L. 150 years of the Annual Report of the Chief Medical Officer: onthe state of public health 2008. London: Department of Health.

4. Tzschentke TM, Christoph T, Kögel B, et al. (-)(1R,2R)-3-(3-Dimethylamino-1-ethyl-2-methyl-propyl)-phenol hydrochloride (tapentadol $\mathrm{HCl}$ ): a novel $\mu$-opioid receptor agonist/norepinephrine reuptake inhibitor with broad-spectrum analgesic properties. J Pharmacol Exp Ther. 2007;323:265-76.
5. Mandala M, Moro C, Labianca R, et al. Optimizing use of opiates in the management of cancer pain. Ther Clin Risk Manag. 2006;2:447-53.

6. Cherny N, Ripamonti C, Pereira J, et al. Strategies to manage the adverse effects of oral morphine: an evidence-based report. J Clin Oncol. 2001;19:2542-54.

7. Lange B, Kuperwasser B, Okamoto A, et al. Efficacy and safety of tapentadol prolonged release for chronic osteoarthritis pain and low back pain. Adv Ther. 2010;27:381-99.

8. Wild J, Grond S, Kuperwasser B, McCann B, Steup A, Rauschkol C. Long-term safety and tolerability of tapentadol extended release for the management of chronic low back pain or osteoarthritis pain. Pain Pract. 2010;10:416-27.

9. Imanaka $\mathrm{K}$, Tominaga $\mathrm{Y}$, Etropolski $\mathrm{M}$, Ohashi $\mathrm{H}$, Hirose K, Matsumura T. Ready conversion of patients with well-controlled, moderate to severe, chronic malignant tumor-related pain on other opioids to tapentadol extended release. Clin Drug Investig. 2014;34:501-11.

10. Kress HG, Koch ED, Kosturski H, et al. Tapentadol prolonged release for managing moderate to severe, chronic malignant tumor-related pain. Pain Phys. 2014;17:329-43.

11. Curtis L, Burns A (2016) Unit costs of health and social care 2016. Canterbury: Personal Social Services Research Unit, University of Kent.

12. Health \& Social Care Information Centre. HRG4 2016/17 Local Payment Grouper. National Casemix Office, Winchester, UK. https://digital.nhs.uk/ National-casemix-office/downloads-groupers-andtools. Accessed 20 Oct 2018.

13. NHS National Tariff Payment System 2016/17. https://www.gov.uk/government/publications/nhsnational-tariff-payment-system-201617. Accessed 20 Oct 2018.

14. Buynak R, Shapiro DY, Okamoto A, et al. Efficacy and safety of tapentadol extended release for the management of chronic low back pain: results of a prospective, randomized, double-blind, placeboand active-controlled phase III study. Expert Opin Pharmacother. 2010;11:1787-804.

15. Afilalo M, Kuperwasser B, Kelly K, et al. Efficacy and safety of tapentadol extended release (ER) for chronic pain due to osteoarthritis of the knee: results of a phase 3 study. Pain Pract. 2009;9:159.

16. Hartrick C, Van Hove I, Stegmann JU, Oh C, Upmalis D. Efficacy and tolerability of tapentadol immediate release and oxycodone $\mathrm{HCl}$ immediate 
release in patients awaiting primary joint replacement surgery for end-stage joint disease: a 10-day, phase III, randomized, double-blind, active- and placebo-controlled study. Clin Ther. 2009;31:260-71.

17. Stegmann JU, Weber H, Steup A, Okamoto A, Upmalis D, Daniels S. The efficacy and tolerability of multiple-dose tapentadol immediate release for the relief of acute pain following orthopedic (bunionectomy) surgery. Curr Med Res Opin. 2008;24:3185-96.

18. Lin J, Chow W, Kim MS, Rupnow MF. Real-world treatment pattern and outcomes among patients who took tapentadol IR or oxycodone IR. J Med Econ. 2013;16:685-90.

19. Ikenberg R, Hertel N, Moore RA, et al. Cost-effectiveness of tapentadol prolonged release compared with oxycodone controlled release in the UK in patients with severe non-malignant chronic pain who failed 1st line treatment with morphine. J Med Econ. 2012;15:724-36.

20. Obradovic M, Ikenberg R, Hertel N, Antoñanzas F, Gálvez R, Liedgens H. Cost-effectiveness of tapentadol in severe chronic pain in Spain: a cost analysis of data from RCTs. Clin Ther. 2012;34:926-43.

21. Merchant S, Noe LL, Howe A, et al. Budget impact analysis of tapentadol extended release for the treatment of moderate to severe chronic noncancer pain. Clin Ther. 2013;35:659-72.

22. Bell T, Panchal S, Miaskowski C, Bolge S, Milanova $\mathrm{T}$, Williamson R. The prevalence, severity, and impact of opioid-induced bowel dysfunction: results of a US and European patient survey (probe 1). Pain Med. 2009;10:35-42. 